\title{
UNA METODOLOGÍA PARA DESARROLLAR Y EVALUAR LA USABILIDAD DE ENTORNOS VIRTUALES BASADOS EN AUDIO PARA EL APRENDIZAJE Y LA COGNICIÓN DE USUARIOS CIEGOS
}

\author{
(A METHODOLOGY FOR DEVELOPING AND EVALUATING THE USABILITY OF AUDIO-BASED \\ VIRTUAL ENVIRONMENTS FOR LEARNING AND COGNITION OF BLIND PEOPLE)
}

\author{
Jaime Sánchez \\ Universidad de Chile (Chile)
}

\section{RESUMEN}

Durante la última década se ha desarrollado un trabajo pionero con el uso de entornos virtuales basados en sonido para el desarrollo de la cognición y el aprendizaje en usuarios ciegos. Este artículo describe este innovador trabajo para el aprendizaje de usuarios ciegos sin pistas visuales. Durante más de una década hemos puesto en marcha la investigación cualitativa y cuantitativa sobre el aprendizaje y la cognición con sonido 3D y con el uso de tareas cognitivas para usuarios ciegos. Los resultados de la investigación revelan una real contribución del entorno virtual basado en sonido y de las tareas cognitivas en el aprendizaje y la cognición en usuarios ciegos. El sonido espacial puede ayudar a mejorar y ejercitar la memoria, la percepción háptica, las estructuras cognitivas temporales y espaciales, la orientación y movilidad, el aprendizaje de las matemáticas y ciencias, y la resolución de problemas. Un resultado importante y crucial de esta investigación ha sido la transferencia de este aprendizaje a situaciones de la vida real. Esto nos ha llevado a confirmar la hipótesis de que los entornos virtuales basados en sonido pueden ser usados para construir significado y desarrollar el intelecto en los usuarios ciegos.

Palabras clave: Interacción humano-computador, entornos de aprendizaje interactivo, personas ciegas, diseño y desarrollo de entornos virtuales

\begin{abstract}
During the last decade, a pioneering work on sound-based virtual environments for developing cognition and learning in blind users has been implemented. This paper describes fully this innovative work on blind user's learning and cognition without visual cues. For more than a decade we have implemented qualitative and quantitative research about learning and cognition using 3D sound interactive virtual environments and cognitive tasks with blind users. Research results reveal a real contribution of sound-based virtual environment and specific cognitive tasks to learning and cognition
\end{abstract}


in blind children. Spatial sound can help to improve and rehearse memory, haptic perception, tempospatial cognitive structures, mobility and orientation, mathematics and science learning, and problem solving. A relevant and critical result of this research has been the transfer of virtual learning to real life learning situations. All of this has leaded us to confirm the hypothesis that sound-based virtual environments can be used to construct meaning and develop the intellect of blind users.

Keywords: Human-computer interface, interactive learning environments, blind people, virtual environment design and development.

Los usuarios ciegos necesitan tener más oportunidades para la exploración táctil, para usar su memoria y para desarrollar la discriminación de sonido y las habilidades para la localización de objetos. La ubicación de objetos de audio en entornos virtuales permite la discriminación de las fuentes de sonido. Además, permite a los usuarios reconocer los atributos de los sonidos y su significado [6]. En este sentido, los usuarios ciegos deben tener al menos las mismas posibilidades de acceso a la tecnología digital que tienen los usuarios videntes, ya que esto abre potenciales puertas para el aprendizaje, la comunicación y la accesibilidad.

¿Cómo podemos ofrecer mejores herramientas de aprendizaje mediante el uso de la tecnología a los usuarios ciegos? Por un lado, un entorno virtual se define para apoyar un aprendizaje en el lugar donde los estudiantes interactúan y participan de varias actividades (Ritzema, 2008). Por otro lado, el sonido espacial permite localizar puntos dentro de un entorno virtual (Amandine, Katz, Blum, Jacquemin, Denis, 2005; Heuten, Wichmann, Boll, 2006; White, Fitzpatrick, McAllister, 2008). Este estímulo sonoro trata de dar a los usuarios ciegos un entorno lúdico en el que se pueden desarrollar habilidades cognitivas necesarias para sus actividades diarias, como por ejemplo, un problema a resolver. Si combinamos estos dos aspectos, se obtiene una poderosa herramienta para el aprendizaje y el trabajo cognitivo de los usuarios ciegos.

Frauenberger et al. (2004) presentan un prototipo de interfaz en que la interacción visual de menús, entrada de texto y diálogos se transmite a usuarios no videntes por medio de audio espacial. Proponen que el uso de audio espacial es capaz de proveer acceso a tecnologías actuales de una manera eficiente a las personas con discapacidad visual. Ramloll et al. (2001) investigaron soluciones a la exploración de datos tabulados por medio de sonido hablado y no hablado. Sus conclusiones señalan que el uso de sonido no hablado disminuye la carga cognitiva del usuario, permitiendo orientar su trabajo a resolver tareas exitosamente. 
Durante más de una década, un equipo de investigación ha desarrollado entornos virtuales basados en audio que proporcionan información y colaboran en nuevas formas de aprendizaje (Sánchez, Elías, 2007; Sánchez, Flores, 2005; Sánchez, Hassler, 2007; Sánchez, Lumbreras, 1999). Varias habilidades cognitivas han sido estimuladas y desarrolladas con el uso de entornos virtuales basados en audio: estructura espacial, memoria abstracta, memoria espacial, memoria de corto plazo, percepción háptica, colaboración, resolución de problemas, habilidades matemáticas, orientación y movilidad, estructuras cognitivas tempoespaciales, habilidades de lenguaje, navegación compleja, aprendizaje de la ciencia y programación computacional.

En este capítulo presentamos una metodología para desarrollar y evaluar la usabilidad de entornos basados en audio para el aprendizaje y la cognición de usuarios ciegos, basada en más de una década de investigación y desarrollo.

\section{METODOLOGÍA}

La metodología que se describe aquí se basa en un diseño centrado en el usuario ciego. El usuario es el actor principal en el diseño y desarrollo de todas las tareas y actividades del entorno virtual. La idea es crear rápidamente prototipos sucesivos y así minimizar los errores y los resultados no deseados. En este contexto se debe tener en cuenta que para crear una aplicación para usuarios ciegos, no basta con cerrar los ojos y ponernos en el lugar del usuario. La mayoría de los elementos de interfaces e interacción que conocemos y utilizamos a diario son inútiles para los usuarios ciegos.

El desarrollo de un entorno basado en audio debe ser conducido por un proceso validado que contiene cuatro etapas específicas y definidas: análisis, diseño, implementación y validación (Figura 1). 


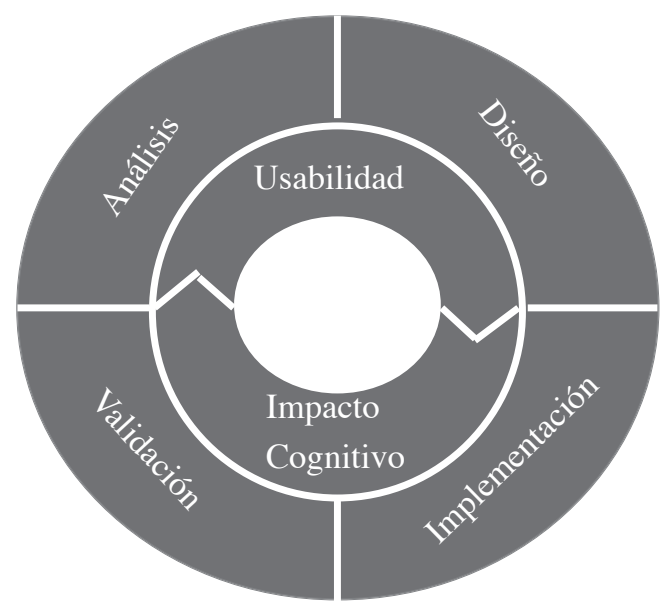

Figura 1. Modelo del proceso de desarrollo de entornos virtuales basados en audio

- Análisis. En esta primera etapa las habilidades cognitivas a desarrollar son definidas por medio del tipo de entorno virtual y las metáforas que se utilizarán. Junto con esto, se define la tecnología y se realiza un estudio del estado del arte de la tecnología y la solución que se desarrollará.

- Diseño. El trabajo es realizado en dos niveles: entorno virtual y tareas cognitivas. Los requisitos del entorno son especificados en los storyboards, guiones, framework de desarrollo y otros aspectos, en conjunto con los resultados obtenidos en la usabilidad. Las tareas cognitivas permiten un trabajo concreto, los usuarios realizan actividades concretas de representación y modelamiento después de interactuar con el entorno basado en audio. Los objetivos y los procedimientos de estas tareas están estandarizados, por lo que es posible repetir la experiencia varias veces con usuarios de diferentes ámbitos y contextos. El entorno y las tareas cognitivas permiten crear un entorno de aprendizaje enriquecido para las personas ciegas.

- Implementación. El desarrollo del entorno sigue un diseño centrado en el usuario, en el que la participación y el feedback de los usuarios son factores clave para la producción rápida de prototipos continuos y progresivos. Este modo de desarrollo permite la evaluación de muchos prototipos en el tiempo, asegurando un producto útil y funcional para los usuarios finales. 
En términos del aspecto cognitivo, si bien todo proceso de evaluación puede y debe llevarse a cabo en diferentes momentos del proceso educativo, al momento de establecer la evaluación de usuario con ceguera o deficiencia visual, es necesario tener claro que de la mayoría de las pruebas estandarizadas que hoy día existen en las distintas áreas de la educación y aquellas complementarias a esta, están diseñadas y elaboradas para personas videntes sin necesidades especificas aparentes. A partir de esta situación surge la necesidad de adaptar la prueba cognitiva requerida para cada caso, considerando aspectos como: grado de visión actual, edad de inicio de la pérdida visual, forma de inicio de la pérdida y evolución de la visión actual. De esta forma, las técnicas de evaluación psicopedagógicas en personas con deficiencia visual son prácticamente las mismas que empleamos en videntes. Sin embargo, existen peculiaridades metodológicas derivadas de la aplicación a personas con deficiencia visual, las que deben incluir las mismas áreas que para personas videntes, eliminando algunos aspectos o ampliando otros específicos del déficit visual (Hall, Scholl, Swallow, 1986). Además, en esta etapa se construye todo el material concreto que se utiliza en las tareas cognitivas.

- Validación. Para validar el entorno, se realizan evaluaciones de usabilidad y cognición. Administrar las evaluaciones de usabilidad a usuarios y a expertos durante y después del diseño del entorno es crítico para generar un producto final de entorno virtual usable y efectivo. Proponemos un conjunto de instrumentos de evaluación de usabilidad claves, tales como: Pauta de Evaluación de Prototipo, Pauta de Evaluación Heurística, Pauta de Evaluación de Usuario Final, Pauta de Entendimiento de Software, Pauta del Facilitador y AudioGram (software para el análisis por medio del uso de la técnica logging actual use).

El propósito de la evaluación cognitiva es determinar si la combinación de entornos virtuales y tareas cognitivas contribuye al desarrollo y aprendizaje de las habilidades cognitivas estudiadas que se definen previamente durante la etapa de análisis (navegación, espacialidad, temporalidad, resolución de problemas, percepción háptica, memoria espacial y abstracta, y pensamiento científico). Los instrumentos utilizados durante la evaluación cognitiva son tareas cognitivas y test cognitivos. Para medir el impacto real del entorno en el aprendizaje generalmente se aplica un diseño de investigación consistente en: pre-test, interacción con el entorno, resolución de tareas cognitivas (modelamiento y representación) y post-test. 


\section{INSTRUMENTOS DE EVALUACIÓN}

El modelo mental es diferente para todos y cada ser humano, pero existen similitudes entre las personas con vivencias similares, las culturas y el conocimiento. Tanto los inmigrantes como los nativos digitales poseen un modelo mental sobre el uso de la tecnología de una forma intuitiva y acceso a la información sin mayores dificultades. Para los usuarios con discapacidad visual la manera de dar forma, solicitar y percibir el mundo son completamente diferentes a los usuarios videntes, lo que genera un modelo mental diferente. Este es sin duda el problema más relevante que estos usuarios tienen que lidiar con el uso de las nuevas tecnologías. Las interfaces no están diseñadas o pensadas para ellos por lo que no es suficiente darles acceso por medio de lectores de pantalla o de procesos.

A lo largo de la investigación, se han utilizado los siguientes instrumentos claves para una correcta evaluación de usabilidad de un entorno basado en sonido para usuarios con discapacidad visual:

- Pauta de Evaluación de Prototipo. Con este instrumento se prueban las primeras interfaces del entorno virtual e interacciones asociadas. En esta etapa se introduce al usuario en el contexto del entorno, para que interactúe de forma intuitiva con las interfaces diseñadas. Esta pauta se elabora con el fin de evaluar los íconos gráficos y auditivos del entorno, y es aplicada en la etapa inicial de diseño de la aplicación. Los usuarios son consultados acerca de su percepción de los diferentes sonidos creados, así como de ciertas imágenes de alto contraste. El tipo de respuesta está asociada a una escala de reconocimiento, existiendo la posibilidad de registrar observaciones.

- Pauta de Evaluación Heurística. Esta pauta consta de ciertos criterios que deben ser evaluados por entre 3 y 5 evaluadores que sean expertos en los conceptos de interfaces y entorno para usuarios con discapacidad visual. La pauta que se utiliza ha sido diseñada en base a las reglas de oro de Shneiderman (2004) y las heurísticas de usabilidad de Nielsen (2007). Hay 12 heurísticas que consideran el estado de la visibilidad del entorno, equilibrio entre el sistema y el mundo real, control de usuario y su libertad de uso, normas y consistencia, prevención de errores, reconocer sobre recordar, flexibilidad y eficiencia de uso, estética y minimalista, ayuda a los usuarios a reconocer, diagnóstico y recuperación de errores, ayuda y documentación, diseño de contenidos, y velocidad y medios. La evaluación se realiza por medio de una escala de apreciación de 5 puntos, que abarca desde muy de acuerdo hasta muy en desacuerdo. 
- Pauta de Evaluación de Usuario Final. Evaluación que realizan los usuarios finales del entorno, correspondiente a un conjunto de preguntas evaluadas según una escala de apreciación. Esta evaluación considera aspectos como la motivación, uso e identificación de los sonidos del entorno.

- Pauta de Entendimiento del Software. Esta prueba consiste en un cuestionario con 10 preguntas abiertas para evaluar la comprensibilidad de los problemas y las tareas planteadas y relacionadas con elementos de la interfaz, como instrucciones, sonidos, señales de sonido, voz, problemas de navegación, y estrategias para encontrar pistas ocultas.

- Pauta del Facilitador. La evaluación fue diseñada teniendo en cuenta a los profesores facilitadores como los usuarios finales cuando llevan a cabo la interacción del niño con el entorno virtual. En consecuencia, reunimos la información que no es posible obtener en las otras evaluaciones. Además de la experiencia con la tecnología, los facilitadores estuvieron presentes durante las interacciones de los usuarios con el entorno, obteniendo así conocimientos y puntos de vista únicos de la interacción de los usuarios.

- AudioGram. Existen diversos aspectos que pueden ser evaluados en la interacción con un entorno virtual. Algunos de ellos han sido mencionados y corresponden a información obtenida mediante pautas de evaluación de usabilidad con usuarios finales. Otro método considera el registro del uso intensivo del mundo virtual, y cuya información relevada no es fácilmente identificable mediante la observación o la aplicación de pautas de evaluación de la usabilidad.

Por cada juego en un mundo virtual se genera un archivo de log para su posterior análisis. Estos archivos contienen toda la información necesaria para el análisis de los datos. Los archivos contienen la estructura del mundo virtual, acciones realizadas por el aprendiz, tiempo en que se realizó la acción y posición dentro del mundo virtual en función del tiempo (Figura 2A).

Para evaluar los resultados del uso intensivo del mundo virtual no observable, se diseñó y desarrolló AudioGram, una herramienta que ayuda al análisis de datos cuantitativos que se obtienen durante la interacción del usuario con el mundo virtual. 
AudioGram otorga la posibilidad de observar algunas variables a largo plazo, como son los tiempos de juego, la cantidad de acciones realizadas, la detección de los distintos errores y choques en el tiempo (Figura $2 \mathrm{C}$ ). Gracias a esto, es posible analizar el aprendizaje del usuario al enfrentar las mismas situaciones. La obtención de los datos se hace por medio de cargar en el entorno virtual todos los logs o registros de los juegos realizados por un usuario. Una vez realizado esto, los datos son graficados por el entorno con el fin de mostrar las variaciones de comportamiento en el juego de un sujeto a través del tiempo. El entorno es flexible permitiendo seleccionar el tipo de variables se desea observar. En el eje horizontal del gráfico aparece el número que representa a cada juego en particular y es posible seleccionar cualquier juego en específico y luego desplegar un análisis detallado en otra ventana (Figura 2B).

Gracias al uso de AudioGram se pueden detectar aspectos en la interacción imposible de lograr por medio de instrumentos clásicos de evaluación. A través de la duplicación de toda la interacción realizada por el usuario en el juego, el entorno permite conocer los pasos y conductas del usuario, tiempos de demora, errores y logros.
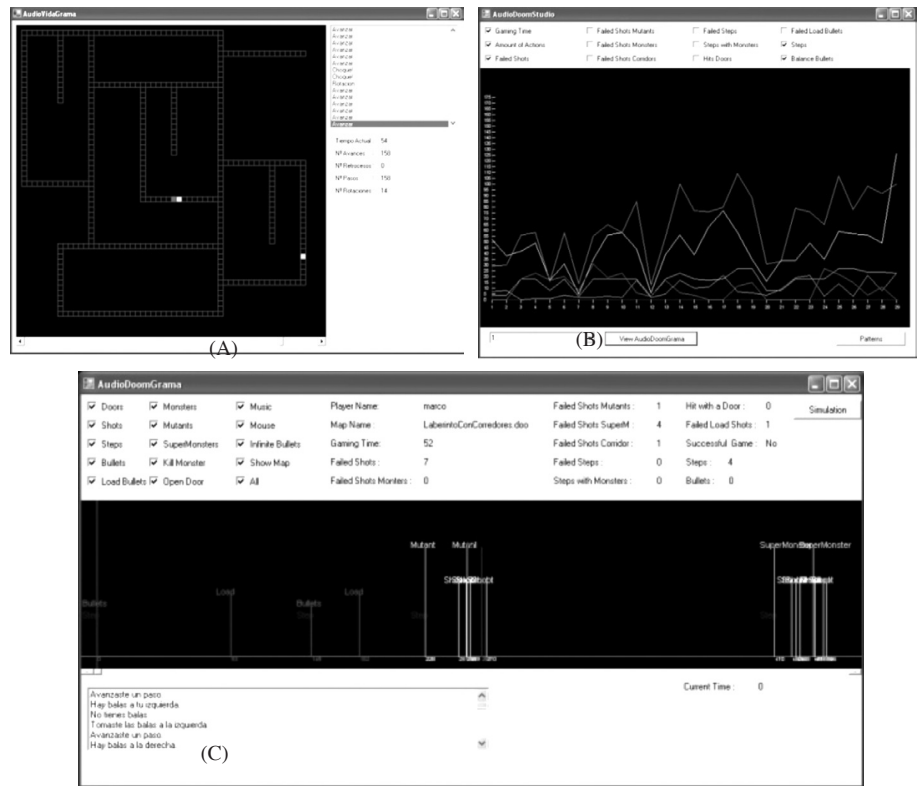

Figura 2. (A) Mapa de interacción. (B) Gráfica del juego. (C) Detalle de las acciones del juego 
Los instrumentos que se utilizan en la evaluación cognitiva corresponden a:

- Tareas Cognitivas. El trabajo en conjunto con los entornos diseñados para usuarios con discapacidad visual y las tareas cognitivas utilizando material concreto, persiguen la estimulación de la percepción y formación abstracta de elementos y objetos. Los usuarios entienden más fácilmente cuando estudian primero en material concreto los elementos y objetos que luego trabajan virtualmente en el entorno. La interacción en paralelo y de forma coordinada de ambas metodologías es una ventaja para los usuarios.

La interacción durante el desarrollo de estas tareas se produce entre los usuarios, el profesor facilitador, los métodos de aprendizaje y la tecnología. El usuario atiende instrucciones, verbaliza acciones a realizar para interactuar efectivamente con el material concreto, aplica con el material concreto los conceptos aprendidos y utilizados virtualmente en el entorno, participa en un focus group y responde las pautas de evaluación. El profesor facilitador debe diseñar y explicar las actividades de aprendizaje, favorecer la interacción del usuario con el entorno por medio de variadas estrategias metodológicas, diseñar y aplicar pautas de evaluación y realizar registro anecdótico. El método de aprendizaje permite la integración de información proveniente de un medio audiovisual, ejercita el desarrollo de la atención, favorece el uso de memoria y beneficia la expresión verbal del usuario, permitiendo destacar los aspectos positivos del entorno. Finalmente, la tecnología asiste el aprendizaje a través de la utilización de herramientas computacionales que apoyen al proceso cognitivo que estimula el entorno.

Los usuarios utilizan material concreto, macrotipo y Braille. La idea es que los usuarios participen de actividades con el uso de estos materiales que están relacionados con el contenido que desarrolla el entorno y de esta manera complementan su trabajo cognitivo. Para efectos de identificar si existen resultados significativos es deseable trabajar con diseños de investigación con dos grupos, control y experimental. El grupo control trabaja única y exclusivamente con el entorno, mientras que el grupo experimental trabaja con el entorno y las tareas cognitivas. Una de las ideas que se busca con el desarrollo de estas tareas es que los usuarios desarrollen en el mundo real actividades similares a las que se presentan en el mundo virtual, buscando la transferencia de virtual a lo real. 
(A)

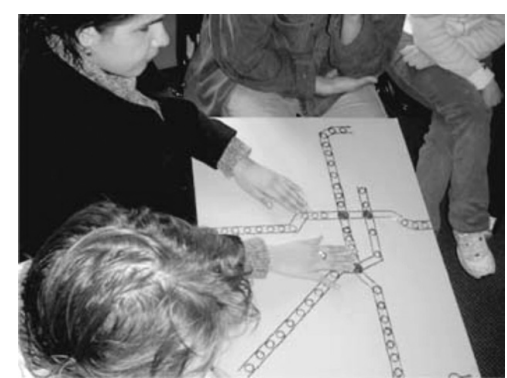

(B)

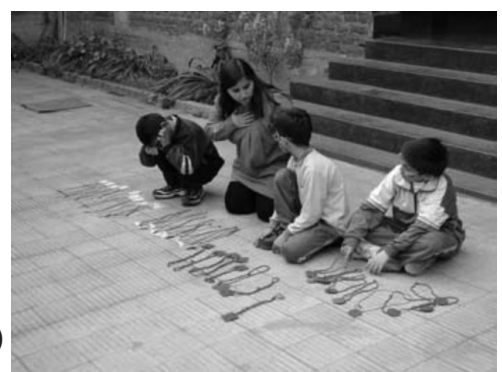

(C)

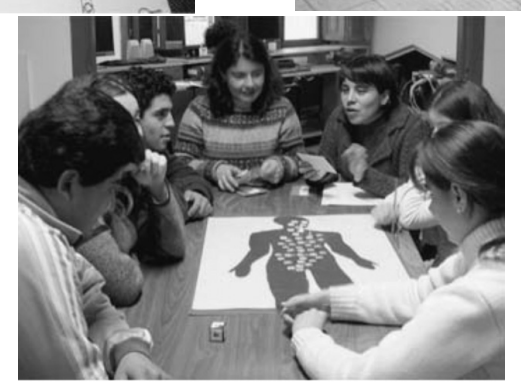

Figura 3. Usuarios trabajando tareas cognitivas con material concreto para el entorno virtual (A) AudioMetro, (B) AudioMath y (C) AudioMUD

- Test Cognitivos. Según sea el estudio cognitivo que se realiza se utilizan diferentes test validados para medir aprendizaje y habilidades cognitivas de dominio general y específico. Así por ejemplo, para evaluar el impacto del entorno basado en audio en el aprendizaje y puesta en práctica de conceptos matemáticos como suma, resta y cardinalidad es posible utilizar el Manual de la Prueba de Precálculo de Milicic y Smith (1997). Junto con este test, para efectos de conocer el impacto del conocimiento matemático, utilizamos el test de Benton y Luria que ha sido adaptado para usuarios no videntes por Chadwick y Fuentes (1980). La prueba de precálculo fue propuesta para medir el desarrollo matemático de usuarios de primer grado. El test de conocimiento matemático permite medir la capacidad de entender de forma oral y escrita los números, la habilidad de hacer cálculos mentales y escritos, la habilidad de contar series numéricas y elementos gráficos, y la habilidad de razonamiento matemático.

Para efectos de evaluar el impacto del uso del entorno que ayuda a la orientación y movilidad utilizamos el test Specific Route Displacement. Para evaluar el desempeño y uso de habilidades de orientación y movilidad de un usuario al utilizar una ruta recurrimos a escalas de apreciación, especialmente desarrolladas para este propósito. 


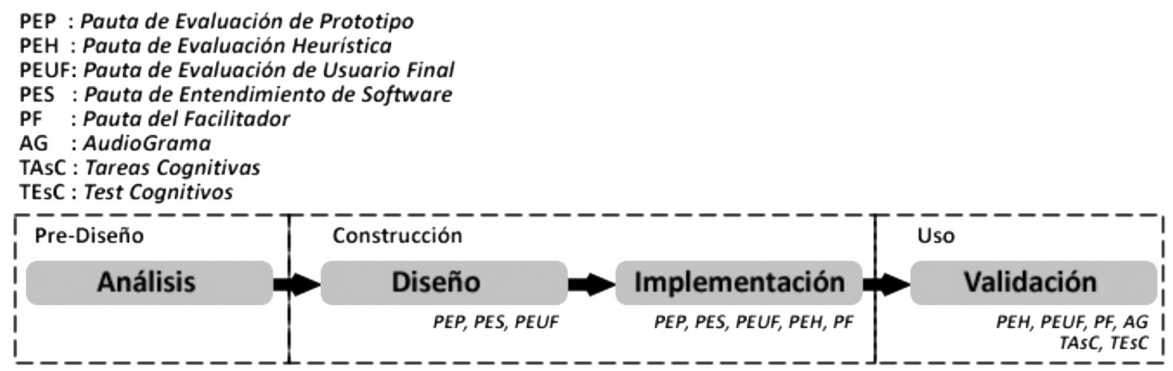

Figura 4. Uso de los instrumentos de evaluación durante el diseño, construcción y uso del entorno virtual

\section{ENTORNOS VIRTUALES BASADOS EN AUDIO}

Durante la última década se ha realizado un trabajo pionero en el uso de entornos virtuales basados en sonido para el desarrollo de la cognición y el aprendizaje de usuarios no videntes. Se han diseñado diversos entornos virtuales basados en audio, tareas cognitivas y métodos de usabilidad para desarrollar y ejercitar procesos cognitivos, tales como relaciones espacio-temporales, memoria, resolución de problemas, movilidad, orientación, abstracción espacial, percepción háptica e integración cognitiva.

Usando la metodología antes descrita, una variedad de entornos virtuales ha sido creado, permitiendo el desarrollo de diferentes habilidades cognitivas (Tabla 1). Cada uno de los entornos que se presentan, han sido evaluados por usuarios no videntes por medio de uso de pautas de usabilidad que hacen que el producto final sea usable y favorezca el desarrollo de una habilidad cognitiva. 


\begin{tabular}{|c|c|c|c|c|c|c|c|c|c|c|}
\hline & 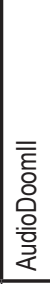 & 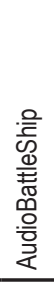 & 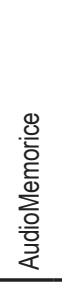 & $\begin{array}{l}\frac{5}{5} \\
\sum_{0}^{\frac{\pi}{0}} \\
\text { 응 } \\
\frac{1}{2}\end{array}$ & 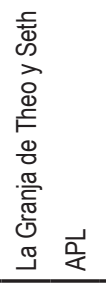 & 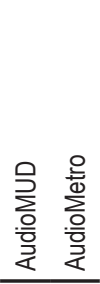 & 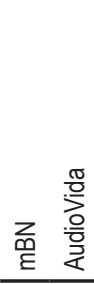 & 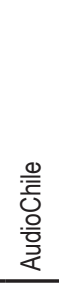 & 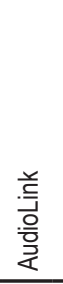 & 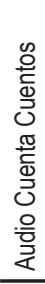 \\
\hline Estructura Espacial & $x$ & & & & & & $\mathrm{X}$ & $\mathrm{x}$ & $x$ & \\
\hline bstracta & & $x$ & & & & & & & & \\
\hline Memoria Espacial & & $x$ & $x$ & $x$ & & & & & & \\
\hline Memoria de Corto Plazo & & & $x$ & $x$ & & & & & & \\
\hline Percepción Háptica & & $x$ & $\mathrm{X}$ & $x$ & & & & $\mathrm{x}$ & & \\
\hline Colaboración & & $\mathrm{x}$ & & & & $\mathrm{X}$ & & & & \\
\hline Resolución de Problemas & & & & & & $\mathrm{x}$ & $x$ & $x$ & $\mathrm{x}$ & \\
\hline Habilidades Matemáticas & & & & $\mathrm{X}$ & & & & & & \\
\hline Orientación y Movilidad & $x$ & & & & & & $\mathrm{x}$ & $x$ & $\mathrm{x}$ & \\
\hline Estructuras Cognitivas Tempo-Espaciales & $x$ & & & & & & & $x$ & $x$ & \\
\hline Habilidades de Lenguaje & & & & & & & & & & $x$ \\
\hline Navegación Compleja & $\mathrm{x}$ & & & & & & & & & \\
\hline Aprendizaje de la Ciencia & & & & & & & & & $\mathrm{x}$ & \\
\hline Programación & & & & & $\mathrm{x}$ & & & & & \\
\hline
\end{tabular}

Tabla 1. Resumen de las habilidades cognitivas que se desarrollan en cada entorno virtual

- AudioDoom II (Sánchez et al., 2001; Sánchez \& Zúñiga, 2006): entorno virtual basado en audio estéreo. Consiste de un laberinto restringido, un pasillo principal y dos pasillos específicos, donde el usuario puede avanzar y resolver tareas a través de audio. La inmersión en el entorno virtual se produce por medio de la ubicación izquierda, centro y derecha de los objetos dentro del laberinto. Las habilidades cognitivas estudiadas con el uso de este entorno son orientación y movilidad, estructuras espacio-temporales y navegación compleja (Figura 5).
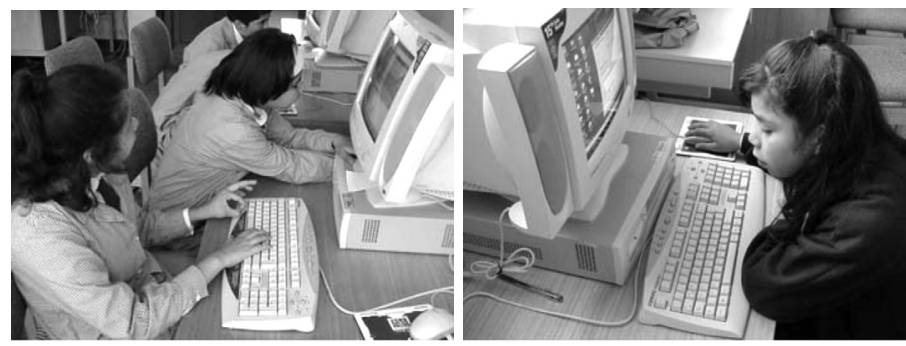

Figura 5. Usuarios jugando el entorno virtual AudioDoomII 
- AudioBattleShip (Sánchez, 2005b; Sánchez et al., 2004): Entorno con interfaz de audio estéreo basado en el tradicional juego de salón, BattleShip. Permite jugar entre usuarios ciegos, ciegos y videntes, y ciegos y máquina. La principal interfaz de entrada es la tablet, a la que se le adhiere una superficie con una grilla de silicona que representa el entorno de juego. Básicamente el audio consiste en ciertas instrucciones verbalizadas a los usuarios, además de sonidos de guerra que se gatillan según los aciertos o desaciertos que tenga el usuario en el juego. Las habilidades que se estimulan en este entorno corresponden a percepción háptica, memoria abstracta, memoria espacial y actividades colaborativas (Figura 6).
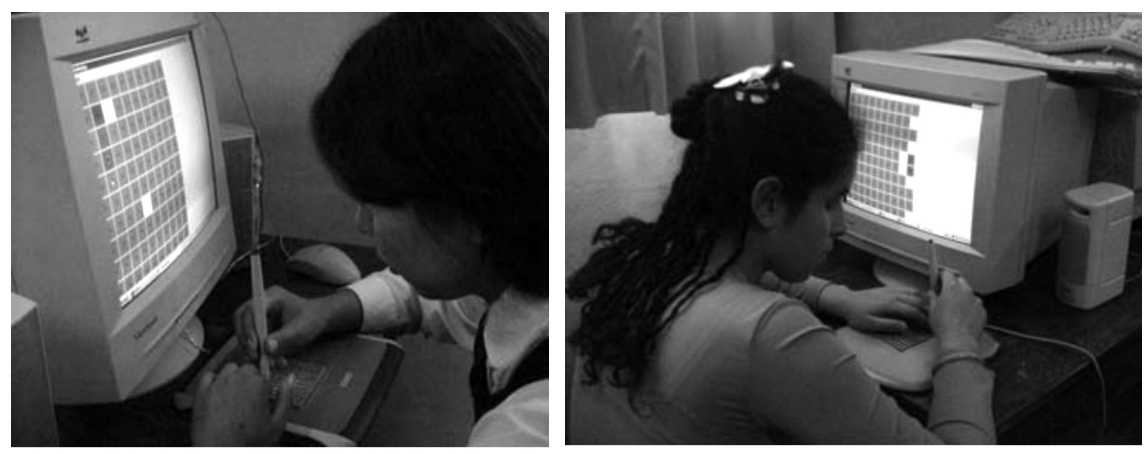

Figura 6. Usuarios jugando el entorno virtual AudioBattleShip

- AudioMemorice (Sánchez \& Flores, 2004): Videojuego basado en el juego de salón, Memorice. El objetivo principal es la estimulación del uso de la memoria de corto plazo. La representación del espacio se hace por medio de notas musicales para cada ubicación en el tablero, reforzado con sonido estéreo. Cada tarjeta que aparece en el juego tiene asociado un sonido que la represente, el que puede ser verbalizado o icónico. Estos sonidos son claves para que los usuarios puedan identificar la información que otorga la tarjeta y con esto poder encontrar los pares correspondientes. El usuario puede interactuar con el entorno por medio del teclado, una tablet con grilla de silicona en la superficie, o bien un joystick con forcefeedback (Figura 7). 

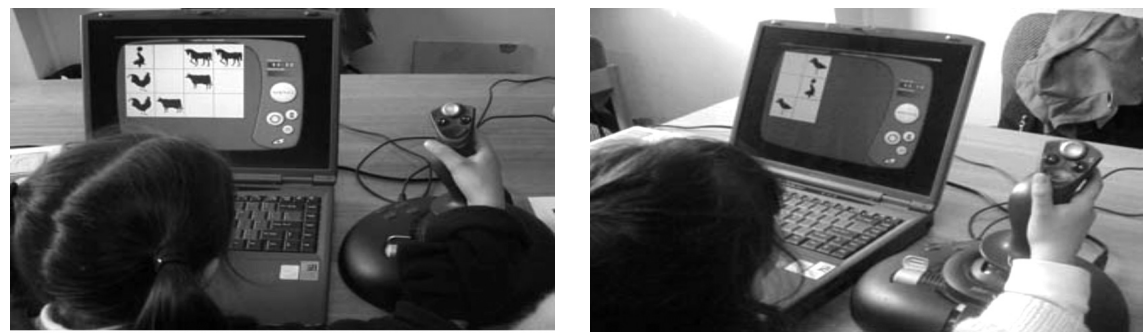

Figura 7. Usuarios ciegos interactuando con el videojuego AudioMemorice

- AudioMath y La Granja de Theo y Seth (Sánchez \& Flores, 2005; Sánchez, 2005a): Entornos virtuales basados en audio estéreo en que el niño aprende y practica conocimientos y operaciones de matemática básica (suma, resta, números ordinales, recta numérica). AudioMath es una instancia de AudioMemorice, por lo que la interacción se realiza de la misma forma, por medio de un joystick o el teclado. En el caso de la Granja de Theo y Seth la interacción es sólo por medio de un teclado. Para la explicación de los conceptos básicos de formación de números se utilizan sonidos lúdicos en conjunto con verbales. Cuando el niño está en una etapa de operatoria, el sonido es principalmente verbal, ocupando sonido lúdico como apoyo a la navegación y la motivación (Figura 8).

(A)

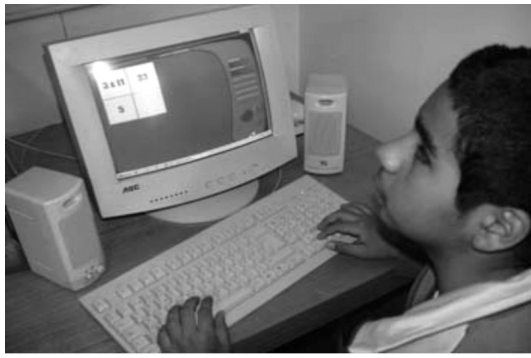

(B)

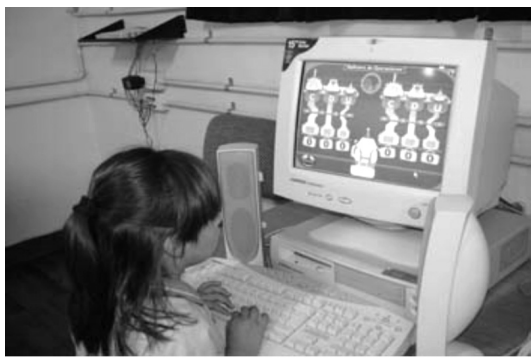

Figura 8. Usuarios ciegos interactuando con (A) AudioMath y (B) La Granja de Theo y Seth 
- APL (Audio Programming Lenguage) (Sánchez \& Aguayo, 2006): APL, es una interfaz basada en sintetizador de voz (Text-to-Speech) para que usuarios no videntes desarrollen habilidades básicas de programación. Los programas desarrollados son ejecutables bajo el mismo entorno de APL. Está orientado a desarrollar habilidades básicas de programación y motivación por la programación computacional (Figura 9).
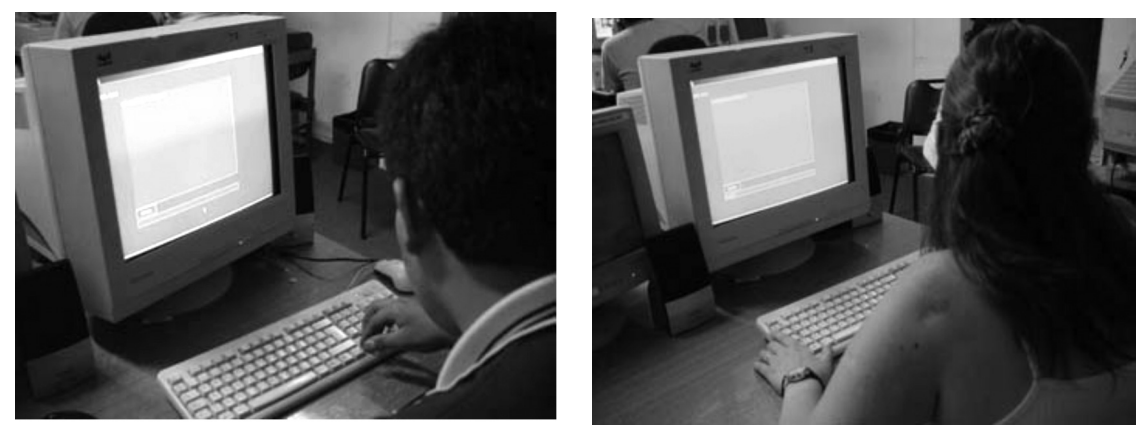

Figura 9. Usuarios ciegos interactuando con APL

- AudioMUD (Sánchez \& Hassler, 2006; Sánchez \& Sáenz, 2007): Entorno virtual basado en el juego de rol MUD (Multi User Dungeon). AudioMUD provee toda la información de forma verbal por medio de voz sintetizada a través de un motor Text-to-Speech. Las habilidades cognitivas estudiadas en este juego son colaboratividad y resolución de problemas en ciencia (Figura 10).
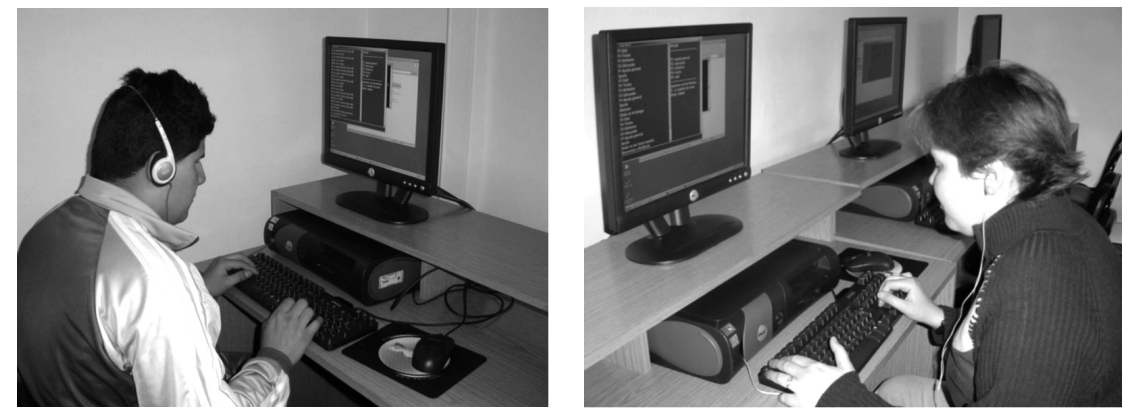

Figura 10. Usuarios ciegos interactuando con el entorno virtual AudioMUD 
- AudioMetro y mBN (mobile Blind Navigation) (Sánchez \& Maureira, 2006): Herramientas basadas en audio estéreo para el desarrollo de habilidades de movilidad y orientación, y resolución de problemas en una red de Metro. AudioMetro funciona en un computador de escritorio y permite preparar un viaje, mientras que $\mathrm{mBN}$ se desarrolló para un dispositivo móvil PocketPC, permitiendo al usuario utilizar el entorno in situ en una estación de Metro. En ambas aplicaciones se utiliza audio estéreo, verbal e icónico. Dada la capacidad de almacenamiento de un dispositivo pocketPC, mBN utiliza un motor Text-toSpeech para los sonidos verbalizados (Figura 11).
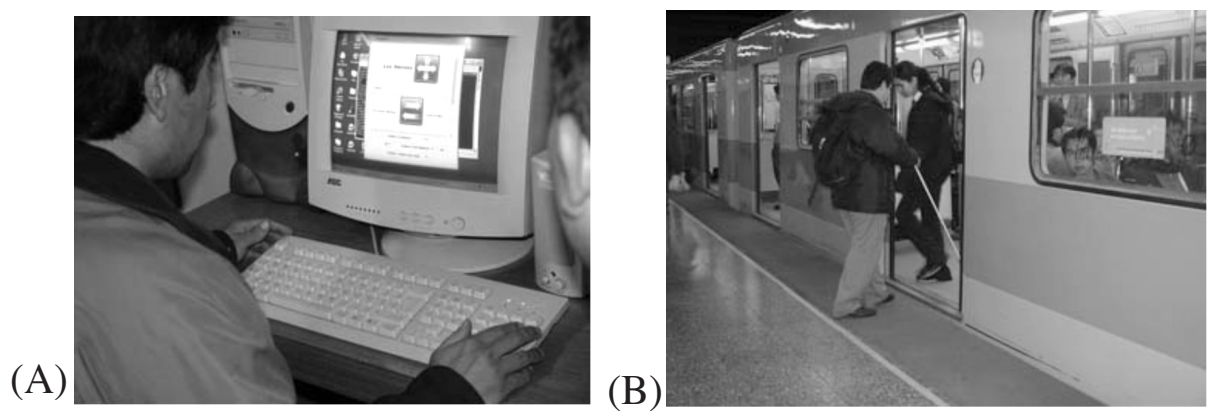

Figura 11. Usuarios ciegos interactuando con (A) AudioMetro y (B) $m B N$

- AudioVida y AudioChile (Sánchez \& Sáenz, 2007): Entornos virtuales basados en audio orientados a la resolución de problemas y a la navegación y orientación de los usuarios en entornos virtuales. En AudioVida diferentes pasillos son descritos por sonidos específicos. La ubicación de los elementos dentro del entorno se especifica por sonido estéreo. En AudioChile, la ubicación en el laberinto es entregada por la variación de la intensidad del sonido en las cuatro orientaciones del personaje. Estas pistas audibles son entregadas por medio de sonido espacial (Figura 12). 
(A)

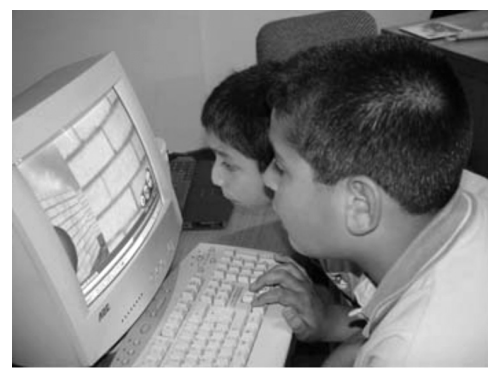

(B)

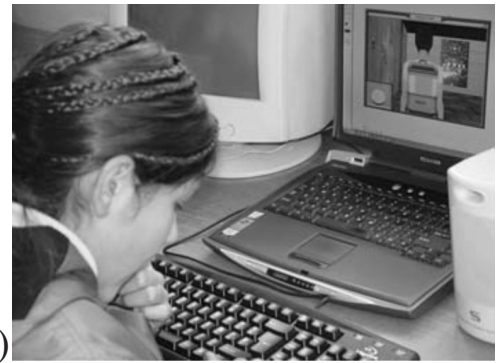

Figura 12. Usuarios ciegos interactuando con (A) AudioVida y (B) AudioChile

- Audio Link (Sánchez \& Elías, 2006): Entorno de sonido espacial en que el usuario navega resolviendo problemas por medio de la interacción con personajes y objetos. En este entorno virtual existen diversos personajes y objetos que son fácilmente reconocibles por los usuarios por medio de sonidos representativos. Los personajes del entorno son trabajados de forma lúdica para lograr una inmersión en la historia. El trabajo se orientó a las habilidades de resolución de problemas, aprendizaje de la ciencia, movilidad y orientación (Figura 13).
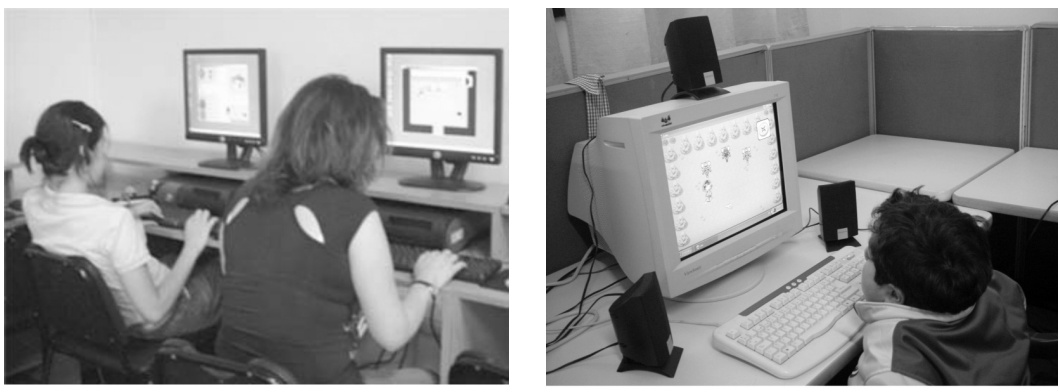

Figura 13. Usuarios ciegos interactuando con AudioLink

- Audio Cuenta Cuentos (Sánchez \& Galaz, 2007): Aplicación móvil para dispositivos PocketPC, basada en texto hablado, para estimular la comprensión lectora de usuarios no videntes. Este entorno intenta suplir un libro, otorgando sus mismas capacidades y formas de uso. El uso de voz sintetizada a través de un motor de Text-to-Speech otorga una forma fácil de agregar libros a la aplicación (Figura 14). 

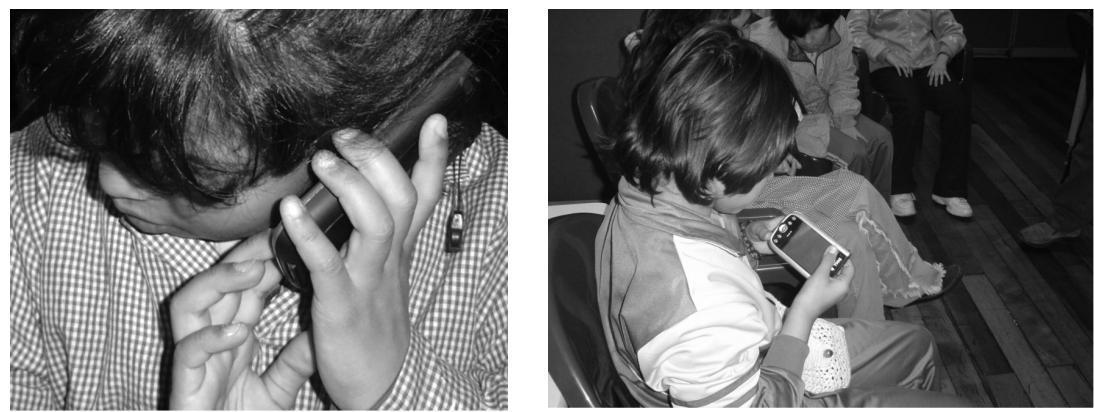

Figura 14. Usuarios ciegos interactuando con AudioCuentaCuentos

\section{INTERACCIÓN DE USUARIOS CIEGOS CON ENTORNOS VIRTUALES}

La interacción de un usuario con un computador se realiza por medio de interfaces de entrada y salida. Por una parte, las interfaces de entrada ampliamente utilizadas por la mayoría de los usuarios son el teclado y el mouse. Por otra parte, las interfaces de salida son gráficas o texto en el monitor y/o papel impreso, y el audio a través de altavoces o audífonos. Interfaces como el mouse están hechas para ser utilizadas visualmente, en la que el usuario debe posicionar el cursor en un área determinada para ejecutar una acción. Por este motivo los usuarios ciegos no pueden utilizar un dispositivo como el mouse y deben utilizar el teclado, que es una interfaz que provee de mayor feedback háptico y permite una interacción mayor. Una de las ideas que hay detrás de los entornos basados en audio desarrollados, es que basamos la interacción en diseños hechos para que el uso del teclado sea natural, y permita al usuario no vidente acceder a todas las acciones provistas por el entorno.

Junto con el teclado existen otras interfaces de entrada que pueden ser utilizadas por un usuario no vidente. Los joysticks proveen de feedback háptico y son un apoyo a la interacción en entornos virtuales, donde el componente háptico apoya la información que se entrega por medio del audio. Acciones como moverse dentro del entorno virtual son mucho más directas y naturales con el uso de un joystick que con el uso de las flechas en un teclado normal. Otra interfaz de apoyo, pero con un uso mucho más específico es la tablet. Por medio de identificar concretamente una grilla ubicada en la superficie de la tablet, el usuario puede identificar casillas y seleccionar la acción que quiere ejecutar. 
Todas estas interfaces son fácilmente encontradas en el mercado común, lo que hace muy accesible el uso de los entornos de parte de los usuarios no videntes más allá del contexto de la investigación, pudiéndolos utilizar en las escuelas o en el hogar.

Como interfaz de salida para los usuarios ciegos se ocupa el audio espacial. Dependiendo del entorno virtual en que se quiera sumergir al usuario, se puede utilizar sonido estéreo, sonido cuadrafónico o superior. Con el sonido cuadrafónico los usuarios logran identificar el origen de las fuentes sonoras, y con ello identificar la ubicación de personajes y objetos dentro del entorno. Otra forma de utilizar el sonido es por medio de diferenciar el audio en el espacio estéreo y utilizar sonido verbalizado.

Un dispositivo que es netamente visual es la PocketPC, principalmente porque funciona bajo la misma idea que un mouse: mirar, identificar, apuntar y seleccionar la acción que se requiere. Para el caso de un dispositivo handheld con sistema touchscreen, esta interacción se realiza por medio de un lápiz stylus. Para los usuarios no videntes, existen dos opciones: 1. Utilizar la botonera que proporciona una PocketPC, pero con número limitado de posibles acciones, reduciéndose en su mayoría a sólo cinco acciones, más el movimiento por la pantalla. 2. Utilizar la pantalla táctil aprovechando su relieve con el resto del dispositivo. Gracias a esto, los usuarios ciegos son capaces de identificar las esquinas, y los puntos medios de la misma. En estas posiciones se colocan botones virtuales que son fácilmente accionados por los usuarios.

En un dispositivo PocketPC la interfaz de salida puede ser sonido verbalizado a través de un motor Text-to-Speech, y sonido monofónico, para no perder el sonido del entorno. Para obtener un sonido espacial se puede utilizar sonido estéreo y simular por medio de máscaras de audio, la ubicación de las fuentes sonoras. La posición frontal izquierda, central y derecha se da sin problemas con audífonos estéreos, mientras que la de los sonidos posteriores se le agrega una máscara, obteniendo la ubicación posterior izquierda, central y derecha.

\section{RESULTADOS DE USABILIDAD}

La importancia de las evaluaciones de usabilidad se reflejan en la afirmación acerca de que el entorno es mucho más fácil de manejar en la versión final que en los primeros prototipos, no sólo por el alto nivel de apropiación que puedan llegar a tener los usuarios, sino porque los entorno en la etapa de desarrollo se van ajustando al modelo mental, las necesidades e intereses de los usuarios finales a través de 
prototipos incrementales gracias a la información obtenida de las evaluaciones de usabilidad que se desarrollan. Las evaluaciones de usabilidad muestran que cuando se diseñan interfaces gráficas los usuarios con visión residual rompen esquemas en cuanto a los patrones establecidos un diseñador de interfaces. Muchas de las guidelines que se siguen para el desarrollo de las interfaces gráficas de usuario o GUIs no tienen aplicabilidad para el caso de usuarios no videntes, principalmente por el modelo mental que tienen asociado. Los usuarios con visión residual presentan dificultades para reconocer ciertos íconos y asociarlos a las acciones para las que fueron diseñados. Como consecuencia, es necesario generar elementos gráficos acordes a la realidad y al modelo mental de los usuarios con visión residual.

El uso de entornos virtuales con sonido espacial permite a los usuarios diferenciar e identificar el sonido envolvente que los ayuda a orientarse espacialmente. Los resultados del estudio de usabilidad de AudioDoomII muestran que los usuarios logran una completa representación del mundo virtual navegado. Los mapas que se presentan en los entornos virtuales AudioChile y AudioVida ayudan a los usuarios a mejorar sus conceptos de lateralidad, de arriba y abajo, representados por el norte y sur. La idea de ubicar a los diferentes objetos por medio de sonido acorde a los cuatro puntos cardinales funcionó satisfactoriamente con los usuarios, permitiéndoles identificar elementos importantes del mundo virtual, como una pared, el borde del bosque y la playa, todos espacios que limitan y ubican al usuario en el entorno. Los usuarios tuvieron una completa comprensión de las estructuras espaciales de los diferentes entornos virtuales diseñados en estos juegos. Esto queda reflejado en la capacidad de los usuarios para representar con material concreto los entornos recorridos.

Los usuarios presentaron una gran motivación y aceptación de los entornos desde las primeras evaluaciones de usabilidad durante el desarrollo hasta las últimas evaluaciones del prototipo final. Los sonidos les ayudaron a captar su atención y a mantenerlos motivados al momento de jugar. Un diseño correcto y apropiado de las interfaces gráficas y de audio produce una satisfacción eficiente y efectiva durante la interacción. Gracias a estas características es posible pensar en agregar mayor complejidad incorporando nuevas misiones, personajes, objetos y elementos. Los usuarios finales siempre desean jugar nuevamente los juegos lo que muestra que la interacción propuesta es atractiva y placentera. En el caso particular de AudioMetro, una de las motivaciones más relevantes para los usuarios es el valor que le otorga la herramienta generando mayor autonomía en la planificación de sus rutas y viajes. Sin el entorno virtual deben acudir a la ayuda de otras personas videntes que le entreguen la información necesaria. En el caso de AudioLink, los usuarios 
demostraron un alto nivel de aceptación y motivación al momento de interactuar con los mini-juegos incluidos.

El sonido siempre debe aportar información útil al usuario y no es un adorno o un complemento como lo puede ser en aplicaciones para videntes. Junto con esto es importante mantener sonidos normalizados y que sean consecuentes con lo que se desea representar. La motivación del uso del entorno se relaciona con los sonidos y el entorno acústico creado, tanto para usuarios con visión residual como para ciegos totales. El uso de las aplicaciones basadas en sonido ha permitido que el aprendiz logre diferenciar e identificar sonidos ambientales que le permiten orientarse espacialmente. También han contribuido a mejorar la lateralidad y los conceptos espaciales de arriba y abajo. Al reconocer y aceptar los sonidos el usuario obtiene un mayor control y navegabilidad a través del entorno virtual.

El modelo mental de todos y cada uno de los seres humanos es distinto, sin embargo existen similitudes entre personas de un mismo lugar, misma cultura o similar nivel de conocimiento. Tanto los inmigrantes como los nativos digitales tienen un modelo de cómo usar la tecnología de una forma intuitiva y acceden a la información sin mayores dificultades. Para los usuarios no videntes la manera de dar forma, ordenar y percibir el mundo es completamente diferente, lo que genera un modelo mental totalmente diferente. Este es el mayor problema que tienen este tipo de usuarios para utilizar nuevas tecnologías, cuyas interfaces no han sido diseñadas para este tipo de usuarios, ni tampoco les otorgan accesibilidad. $\mathrm{Al}$ respecto, se han identificado dos puntos esenciales: 1. Para un vidente existen diversos aspectos de interfaces gráficas que son reconocibles y altamente utilizadas. Usar los mismos aspectos gráficos para usuarios con visión residual no garantiza un buen resultado. A lo largo de la investigación, existen variados sonidos que han sido probados y aceptados por los usuarios no videntes, generando una librería reconocible y asociada a acciones. 2. Una forma clásica de presentar y realizar acciones en un entorno virtual es por medio de menús organizados según una cierta jerarquía. Esta jerarquía permite tener una buena visibilidad del menú, y accesibilidad directa por medio de un puntero. Al utilizar el teclado como medio de interacción con el menú, con o sin visibilidad total de las acciones, el usuario no vidente accede a una acción por vez. Entonces no es necesario priorizar la visibilidad, sino que se debe enfatizar una fácil navegabilidad por las diferentes opciones del menú. Se ha puesto énfasis en que en la presentación de los menús del entorno, estos sean basados en el concepto de listas circulares. La idea consiste en que tiene un inicio, pero no tiene un final, el último elemento del menú está asociado al primero. Cuando se desarrolla una GUI para usuarios videntes es necesario que todos los elementos importantes sean 
visibles y alcanzables a un click del mouse. Los no videntes no necesitan de esta visibilidad ya que pueden acceder a un control a la vez por medio del teclado. Cuando utilizan el teclado la interacción es de un elemento del menú, el que lo recorren hacia delante o hacia atrás.

Es importante proveer de instrucciones claras antes y durante la interacción con el entorno. Estas no deben confundir al usuario sino ser una guía que les permita comprender completamente la tarea que se requiere resolver. A medida que el usuario gana experiencia con el uso de la aplicación estas instrucciones pueden disminuir. El aprendiz con discapacidad visual y sobre todo el ciego total, necesita de muchas pistas e instrucciones que ayuden a una mejor orientación, que deben ser lo más parecido a su entorno real. El diseño apropiado y correcto de las interfaces auditivas y visuales permite que los usuarios ciegos sean capaces de cumplir los objetivos planteados en los entornos virtuales de forma efectiva y eficiente, logrando satisfacción durante el proceso de interacción. Gracias a estas características es posible pensar en agregar mayores complejidades a los entornos virtuales, incorporando nuevas misiones, personajes, objetos y elementos.

Los usuarios con remanente visual tratan siempre de utilizar su reducida visión al momento de interactuar con el entorno. Por este motivo es sumamente importante al momento de crear interfaces gráficas para estos usuarios, utilizar colores de alto contraste que permitan la diferenciación de formas y figuras. Junto con esto se deben utilizar íconos claros y directos que representen adecuadamente la funcionalidad que posee el botón, manteniendo las prestaciones apropiadas.

El uso de dispositivos móviles por parte de usuarios no videntes es posible. Dado que es un dispositivo nuevo para estos usuarios, existen algunas dificultades iniciales para su utilización, las que se ajustan de acuerdo a su uso, adoptando la manera más cómoda para cada usuario. Entre las formas de utilizar el dispositivo, algunos optaron por usar un solo audífono de manera de liberar un oído para escuchar el sonido ambiental y no perder información importante del contexto. Además de esto, la forma de transportar la PocketPC varia, algunos la llevan en su bolsillo, otros colgada al cuello o bien en una mano. En un dispositivo PocketPC la interacción se realiza principalmente por medio de la pantalla táctil, pero también posee cuatro botones de acceso directo más un joystick de navegación. Los usuarios no videntes reconocen táctilmente estos botones y los utilizan sin dificultades, además aprovechan los relieves que se forman entre la pantalla táctil y la pocket para guiarse y así poder ocuparla. Más eficientemente. Particularmente, ellos reconocen muy fácilmente las esquinas y los puntos medios entre una esquina y otra. De esto se saca 
provecho para el diseño de una interfaz que puede asociar acciones en estos puntos o bien según la unión de los mismos. Por el hecho de que se deben ubicar táctilmente para encontrar los espacios de acción, los usuarios utilizan sus dedos como punteros y no utilizan el stylus.

El uso de un joystick con forcefeedback es un excelente apoyo para la interacción. Este dispositivo introduce un nuevo escenario en los entornos virtuales para usuarios ciegos. El joystick háptico puede proveer información y sensaciones táctiles por medio del forcefeedback. Permite otorgar información en conjunto con el audio, evitando la excesiva presencia de sonidos que pudieran saturar e incluso confundir al usuario. Además, estos joystick tienen la cualidad de tener una cantidad apropiada de botones que permiten asociar acciones y así sólo utilizar este dispositivo.

Al utilizar un teclado como interfaz de entrada, se deben utilizar teclas que sean fácilmente reconocibles por los usuarios no videntes, tales como Enter, Espacio, Tabulación y las flechas de movimiento. Todos los teclados QWERTY tienen marcas en las teclas F y J, las que sirven de referencia para identificarlas y utilizar las otras teclas alrededor de estas.

\section{RESULTADOS COGNITIVOS}

Como resultado del uso de entornos basados en sonido virtual como AudioMemorice y AudioBattleShip los usuarios ciegos han sido capaces de entrenar la utilización de la memoria abstracta de corto y largo plazo. Con estos entornos basados en audio se genera la capacidad de desarrollar y ejercitar imágenes mentales del espacio, así como también su percepción háptica y abstracción espacial. Los resultados son favorables y nos permiten establecer que los usuarios participantes han obtenido importantes avances en el uso de su memoria.

Gracias a la utilización de estos entornos, los aprendices pueden lograr un aprendizaje significativo cuando se utiliza el sonido para representar mentalmente el espacio navegado, siendo posible encontrar rutas alternativas, variando su uso y decisiones. Del mismo modo, los usuarios ciegos prestan más atención a las señales de sonido que identifican un determinado lugar, así como utilizan puntos de referencia para orientarse a través del entorno, pudiendo encontrar rutas más eficientes.

Una vez que los usuarios han explorado completamente el entorno virtual estos son capaces de representarlos por medio de modelos utilizando material concreto, 
demostrando ejercitación de la memoria espacial y abstracta. Debido al hecho de que memorizan la cantidad de vueltas y su secuencia para la obtención de una representación de la ruta explorada, la fidelidad de la representación de un mapa virtual o un laberinto depende directamente de la cantidad de vueltas y las veces que debe explorar el espacio. Hemos encontrado que cuando el usuario explora más de 4 veces una ruta determinada con no más de 6 vueltas, la representación final es muy fiel a la original.

Los usuarios que trabajaron con los entornos virtuales de matemáticas en conjunto con tareas cognitivas incrementaron de forma notable sus conocimientos de conceptos básicos y aumentaron su desempeño para resolver las operaciones básicas como suma, resta, multiplicación y división.

Los diferentes contextos y problemas que los usuarios han enfrentado en los entornos virtuales para resolver problemas han permitido la generación de una diversidad de experiencias para que puedan identificar problemas, resolverlos y evaluar el proceso. Los resultados obtenidos de la evaluación del impacto del uso de los entornos virtuales basados en sonido para usuarios con discapacidad visual nos muestran que estos pueden ser capaces de anticipar los problemas, planificar y aplicar diferentes estrategias de resolución de problema y también explicar la estrategia utilizada para resolverlos y a su vez aplicar lo aprendido a otros contextos.

Nuestros estudios muestran una mejora en el comportamiento y desarrollo de habilidades y competencias de los usuarios al utilizar la red de Metro, siendo cada vez más independientes. Los usuarios han obtenido mejoras en áreas como la orientación espacial, la orientación temporo-espacial, la interacción verbal, la autonomía, la selección de rutas, y logros de viaje a través de una ruta planificada. Se ha detectado que es esencial que el usuario tenga puntos de referencia, al menos en la etapa donde se forma un modelo mental del espacio virtual explorado. En definitiva, estos resultados muestran que los usuarios logran una mayor autonomía e independencia en su navegación por el Metro.

Los usuarios ciegos han desarrollado sus habilidades de lectura utilizando AudioCuentaCuentos dando espacio a una forma virtual alternativa a los libros en Braille. La facilidad de ampliar la colección de cuentos permite dar acceso a un mayor número de libros ampliando considerablemente las posibilidades de lectura para estos usuarios. La idea no es sólo utilizar el entorno virtual sino que utilizar la tecnología en conjunto con las experiencias previas, las estrategias de estudio y los hábitos de lectura. 


\section{DISCUSIÓN}

El eje principal del desarrollo y la investigación aquí presentada se ha realizado por medio de interfaces basadas en sonido, en las que el audio se utiliza para representar y transmitir información y conocimiento. No hay que olvidar que la audición es uno de los sentidos capaz de suplir a la vista, en cuanto a su capacidad y calidad de información. Por ello, es importante realizar, durante el desarrollo de estas interfaces, continúas evaluaciones de usabilidad que permitan identificar la cercanía de la interfaz con las necesidades e intereses de los usuarios, sus formas de interactuar y sus modelos mentales.

En general, las herramientas desarrolladas han permitido que los usuarios con discapacidad visual logren diferenciar e identificar sonidos ambientales que le ayudan a orientarse y moverse en los distintos espacios. Además han contribuido a mejorar la lateralidad y conceptos espaciales, tales como arriba, abajo, izquierda y derecha. El sonido siempre ha sido un elemento clave en el uso de los entornos virtuales, siendo el eje principal del desarrollo y de la investigación. A través de la gran variedad de estímulos auditivos, el usuario se mantiene atento y motivado durante la interacción con los entornos, y lo más importante, se logra transmitir información y construir conocimiento.

Las tareas cognitivas son de gran ayuda para los usuarios con discapacidad visual. Las tareas cognitivas potencian el tacto activo. Cuando los materiales utilizados son del apego de los usuarios y basados en experiencias de la vida cotidiana, se potencia el interés por aprender y por explorar. Esto es de importancia para los usuarios con discapacidad visual, ya que en ausencia de visión, siendo ésta parcial o total, conocer por medio del tacto permite construir aprendizajes significativos. Por este motivo los entornos deben ir acompañados del trabajo con tareas cognitivas, para que los aprendizajes logrados por los usuarios sean construidos de manera efectiva y los puedan generalizar, y, de este modo, no caer en la verbalización, conducta típica en usuarios con discapacidad visual.

Las evaluaciones de usabilidad son cruciales para representar de forma correcta la interacción que deben tener los usuarios con los entornos virtuales que se desarrollan. Junto con esto se logran interfaces entendibles y usables, y que funcionan más intuitivamente, evitando tener que memorizar o aprender la forma de interactuar. Los usuarios no videntes aceptan, aprecian y usan los entornos virtuales de forma motivante. Después de diseñar y rediseñar las interfaces de sonido espacial ellos logran representar y navegar sin problemas el espacio navegado. 
El sentido de la audición favorece la percepción de puntos de referencia, la descripción de lugares físicos, el desarrollo del pensamiento abstracto, la habilidad de concentrarse y la creación de esquemas mentales de los espacios físicos. Para lograr que esto sea efectivo es imprescindible considerar trabajar directamente con el usuario en todo el proceso de diseño, desarrollo y evaluación de los entornos virtuales basados en sonido, con su modelo mental, necesidades y formas de pensar y razonar. Esto es lo que denominamos diseño centrado en el usuario ciego.

Los entornos virtuales basados en sonido permiten la generación de diversas y adecuadas experiencias en con la finalidad de que los usuarios no videntes sean capaces de reconocer problemas, resolverlos y evaluar el proceso. El feedback proporcionado por los juegos y las distintas alternativas que ofrecen en cuanto a señales auditivas, obstáculos, y niveles de dificultad permiten una interacción activa, estimulante y motivante en todos los usuarios ciegos.

La navegación por los entornos virtuales no es al azar, los usuarios definen y utilizan diversas estrategias que los ayudan en su recorrido y representación. La principal estrategia utilizada consiste en memorizar secuencias de dirección y comparar las distancias relativas entre puntos clave del recorrido. Esto es interesante ya que revela que la estrategia utilizada en un entorno virtual es muy similar a la estrategia que utilizan en su vida real. Esto ofrece la posibilidad de ocupar este sistema para entrenar a los usuarios ciegos para su movilidad y orientación, transformándose en una poderosa herramienta de integración.

Un dispositivo móvil PocketPC con una interfaz basada en sonido puede reforzar las habilidades para una adecuada comprensión lectora en un niño ciego o con baja visión. Sin embargo, los procesos de comprensión a través de la audición y comprensión de lectura llevan implícitas una serie de habilidades a desarrollar, por lo que el entorno virtual debe ir acompañado de otros recursos y complementado con otras estrategias que consideren por ejemplo: intereses, motivación y hábitos lectores de los aprendices.

Finalmente, los resultados que se presentan como resultado del uso de los entornos que se han desarrollado para usuarios ciegos confirman la teoría que el sentido de audición es capaz de reemplazar a la visión en su capacidad de entregar y percibir información. El hecho de que los usuarios no videntes naveguen einteractúen con objetos y entidades dentro del mundo a través de audio constituye una poderosa metodología para representar y construir conocimiento. 


\section{Agradecimientos}

Este trabajo ha sido financiado en parte por el Fondo Nacional de Ciencia y Tecnología, Fondecyt \#1090352 y el Proyecto CIE-05 Programa de Centros de Educación PBCT-Conicyt.

\section{REFERENCIAS BIBLIOGRÁFICAS}

Amandine, A.; Katz, B.; Blum, A.; Jacquemin, C.; Denis, M. (2005). A Study of Spatial Cognition in an Immersive Virtual Audio Environment: Comparing Blind and Blindfolded Individuals. Proceedings of ICAD 05-Eleventh Meeting of the International Conference on Auditory Display, Limerick, Ireland, July 6-9, (228-235).

Chadwick, M.; Fuentes, M. (1980). Evaluation of Mathematics Knowledge (adaptation of Benton-Luria test), Santiago.

Frauenberger, C.; Putz, V.; Holdrich, R. (2004). Spatial auditory displays a study on the use of virtual audio environments as interfaces for users with visual disabilities. In DAFxo4 Proceedings, Naples, Italy, October 5-8, 7th Int. Conference on Digital Audio Effects (DAFx'04), (384-389).

Hall, A.; Scholl, G. T.; Swallow, R. (1986). Psychoeducational assessment. In: Scholl, T. (Ed.) Foundations of Education for Blind and Visually Handicapped Children and Youth, Theory and Practice. New York: American Foundation for the Blind Inc., 509.

Heuten, W.; Wichmann, D.; Boll, S. (2006). Interactive $3 \mathrm{D}$ sonification for the exploration of city maps. In Proceedings of the 4th Nordic Conference on HumanComputer interaction: Changing Roles (Oslo, Norway, October 14 - 18). In: Mørch, A.; Morgan, K.; Bratteteig, T.; Ghosh, G.; Svanaes, D. (Eds.). NordiCHI '06, vol. 189. ACM, New York, NY, (155164).
Milicic, N.; Schmidt, S. (2003). Manual de la Prueba de Precálculo. Editorial Universitaria, ${ }^{\text {a }}$ Ed., (1-140).

Nielsen, J. (2007). Ten usability heuristics. [en línea] Disponible en: www.useit. com/papers/heuristic/heuristic list. html (consulta 2007, 15 de Julio)

Ramloll, R.; Yu, W.; Riedel, B.; Brewster, S. A. (2001). Using Non-speech Sounds to Improve Access to 2D Tabular Numerical Information for Visually Impaired Users. In Proceedings of BCS IHM-HCI 2001 (Lille, France), Springer, (515-530).

Ritzema, T.; Harris, B. (2008). The use of Second Life for distance education. Journal of Computing Sciences in Colleges. 23, 6, (110-116).

Sánchez, J. (2005a). Training blind children to Develop Mathematics Skills through Audio. CyberPsychology \& Behavior, 8 (4), August, (354-355).

Sánchez, J. (2005b). AudioBattleShip: Blind learners' cognition through sound. International Journal on Disability and Human Development, IJDHD, Vol. 4, No. 4, October-December, (303-309).

Sánchez, J.; Aguayo, F. (2006). APL: Audio Programming Language for Blind Learners. In K. Miesenberger et al. (Eds.). Lecture Notes in Computer Science. LNCS 4061, Springer-Verlag Berlin, Heidelberg. (1334-1341).

Sánchez, J.; Baloian, N.; Hassler, T. (2004). Blind to Sighted Children Interaction through Collaborative Environments. In: Gert-Jan de Vreede et al. (Eds). Lecture 
Notes in Computer Science, LNCS 3198, Springer-Verlag Berlin Heidelberg. (192 $-205)$.

Sánchez, J.; Elías, M. (2006). Blind Children Learning Science through Audio-Based Interactive Software. Proceedings of VII Congreso Internacional de Interacción Persona-Ordenador, Interacción. Noviembre 13-17. Universidad de Castilla-La Mancha, Puertollano (Ciudad Real), España, (591-600).

Sánchez, J.; Flores, H. (2004). Memory enhancement through audio. ACM SIGACCESS Accessibility and Computing archive Issue. (77-78, 24-31).

Sánchez, J.; Flores, H. (2005). AudioMath: Blind children learning mathematics through audio. International Journal on Disability and Human Development, IJDHD, Vol. 4, No. 4, October-December, (311-316).

Sánchez, J.; Galaz, I. (2007). AudioStoryTeller: Enforcing Blind Children Reading Skills. In: Stephanidis, C. (Ed.). Universal Access in HCI, Part III, HCII 2007, Lecture Notes in Computer Science LNCS 4556, 786-795, Springer-Verlag Berlin Heidelberg.

Sánchez, J.; Hassler, T. (2006). AudioMUD: A Multi-User Virtual Environment for Blind People. Proceedings of the $5^{\text {th }}$ International Workshop on Virtual Rehabilitation, IWVR '06. New York, USA . August 29-30, (64-71).

Sánchez, J.; Lumbreras, M.; Cernuzzi, L. (2001). Interactive virtual acoustic environments for blind children: Computing, Usability, and Cognition. Proceedings of ACM CHI, Seattle, Washington, April 2-5. (65-66).

Sánchez, J.; Maureira, E. (2007). Subway Mobility Assistance Tools for Blind Users. In: Stephanidis, C.; Pieper, M. (Eds.). Lecture Notes in Computer Science, LNCS 4397, Springer-Verlag Berlin Heidelberg. (386-404).

Sánchez, J.; Sáenz, M. (2007). Usability of Audio-Based Virtual Environments for Users with Visual Disabilities. Virtual Reality and Human Behavior Symposium, LAVAL Virtual, Laval, France, April 18-22.

Sánchez, J.; Zuñiga, M. (2006). Evaluating the Interaction of Blind Learners with Audio-Based Virtual Environments. Annual Review of CyberTherapy and Telemedicine. Virtual Healing: Designing Reality. Volume 4, (167-173).

Shneiderman, B.; Plaisant, C. (2004). Designing the User Interface: Strategies for Effective Human-Computer Interaction. New York: Addison Wesley.

White, G. R., Fitzpatrick, G., and McAllister, G. (2008). Toward accessible $3 \mathrm{D}$ virtual environments for the blind and visually impaired. In Proceedings of the 3rd international Conference on Digital interactive Media in Entertainment and Arts (Athens, Greece, September 10 - 12). DIMEA '08, vol. 349. ACM, New York, NY, (134-141). 


\section{PERFIL ACADÉMICO Y PROFESIONAL DEL AUTOR}

Jaime Sánchez Recibió los grados académicos de M.A. (1983), M.Sc. (1984), y Ph.D. (1985) por la Universidad de Columbia, Nueva York. Es Profesor Titular de Interacción Persona-Computador en el Departamento de Ciencias de la Computación de la Universidad de Chile. Él ha desarrollado varios entornos virtuales basados en sonido para estimular el desarrollo del aprendizaje y la cognición en niños ciegos. En la actualidad, investiga el uso de dispositivos móviles para ayudar a que los aprendices desarrollen y ejerciten habilidades de resolución de problemas en contextos reales. Sus intereses en investigación incluyen audio y desarrollo cognitivo, métodos de evaluación de usabilidad, aprendizaje basado en juegos y aprendizaje móvil. También es autor de varios libros sobre aprendizaje con tecnología de la información y comunicación.

E-mail: jsanchez@dcc.uchile.cl

DIRECCIÓN DEL AUTOR

Blanco Encalada 2120, Zip Code 2777, Santiago, Chile

Fecha de recepción del artículo: 15/05/10 Fecha de aceptación del artículo: 16/07/10 